\title{
Genetic Modeling of Ras-Induced Human Rhabdomyosarcoma
}

\author{
Corinne M. Linardic ${ }^{\star}, \S$ and Christopher M. Counter $§, \uparrow$ \\ *Department of Pediatrics, Duke University, Durham, NC 27710 \\ §Department of Pharmacology and Cancer Biology, Duke University, Durham, NC 27710 \\ IDepartment of Radiation Oncology, Duke University, Durham, NC 27710
}

\begin{abstract}
Rhabdomyosarcoma is the most common soft tissue sarcoma of childhood and adolescence. Historically, rhabdomyosarcoma has been studied by the manipulation of human cell lines derived from primary rhabdomyosarcoma tumor tissue adapted to grow in culture. Recently, mouse models have been added to the arsenal of tools to study this disease in vivo. However, given the emerging understanding of the genetic variability and mutability of human tumor-derived cell lines, and the existing differences between human and murine tumorigenesis, we sought to uniformly dissect the genetic events required to generate rhabdomyosarcoma from primary human skeletal muscle precursors. To this end, primary human skeletal muscle cells were transformed with defined genetic elements to corrupt the p53, Rb, Myc, telomerase, and Ras pathways, resulting in cells that, when assayed as subcutaneous xenografts in immunocompromised mice, formed tumors indistinguishable at the immunohistochemical level from the embryonal histologic variant of rhabdomyosarcoma. This chapter will discuss the techniques used to transform primary human skeletal muscle cells, the assays used to verify expression of the ectopically expressed genetic elements, and the methods used to evaluate the tumorigenic capacity of the resulting cell lines.
\end{abstract}

\section{Introduction}

Historically, the study of rhabdomyosarcoma has relied on cultured cell lines created from patient-derived tumor samples that have been adapted to grow in vitro. Beginning in the 1980s, several transgenic mouse mutants intended to study the role of specific genes were serendipitously found to develop tumors resembling embryonal rhabdomyosarcoma (Merlino and Helman, 1999;Nanni et al., 2003;Sharp et al., 2002;Tsumura et al., 2006). In 2004, a mouse model for alveolar rhabdomyosarcoma was described (Keller et al., 2004). While both cell line and mouse genetic modeling approaches have been invaluable in dissecting rhabdomyosarcoma, it has become clear that both also have limitations. Cell lines derive from genetically diverse individuals, may accumulate additional mutations with prolonged culturing, and represent the culmination of genetic mis-events that eventually result in cancer, thus limiting the ability to define early and intermediate tumorigenic events. Mouse models accurately reflect murine rhabdomyosarcoma formation, but given the recent observed differences between human and murine tumorigenesis(Hamad et al., 2002;Rangarajan et al., 2004), cannot precisely model the human disease. Efforts were therefore directed towards developing in vitro models in human cells that could dissect the discrete genetic events required to step-wise convert primary human skeletal muscle cells into their transformed counterparts.

Contact information: Corinne M. Linardic: linar001 @ mc.duke.edu; telephone 919-684-3401; fax 919-681-7950; Christopher M. Counter: count004@mc.duke.edu; telephone 919-684-9890; fax 919-684-8958.

Senior and corresponding author: Christopher M. Counter 
The first genetically defined model of human cancer showed that in primary human cells, it was necessary and sufficient to inactivate the $\mathrm{p} 53$ and Rb pathways, upregulate Myc pathways, reactivate telomerase in order to stabilize telomeres, and activate oncogenic Ras in order to provide self-sufficiency in growth signaling and angiogenesis (Hahn et al., 1999;Kendall et al., 2005; Yeh et al., 2004). This was accomplished by the stable serial introduction into primary human cells of four transgenes encoding SV40 large T and small $t$ oncoproteins, the hTERT catalytic subunit of telomerase, and an oncogenic version of the G-protein $\mathrm{H}-\mathrm{Ras}{ }^{\mathrm{G} 12 \mathrm{~V}}$. In this chapter, we will describe the adaptation of this approach towards modeling human embryonal rhabdomyosarcoma(Linardic et al., 2005). Importantly, we have chosen to use primary human skeletal muscle cells as the starting cell type for this approach, as rhabdomyosarcoma is defined by its skeletal muscle features. We have also validated the choice of the four described transgenes by assuring that rhabdomyosarcoma indeed demonstrates lesions in the $\mathrm{p} 53, \mathrm{Rb}$, Myc, telomerase and Ras pathways (Linardic et al., 2005;Xia et al., 2002). Regarding oncogenic H-Ras, although this mutation is not common in human embryonal rhabdomyosarcoma, it adequately represents the inappropriate upregulation of the Ras pathway found in rhabdomyosarcoma, manifest as gain-of-function mutations in proteins that drive Ras signaling, such as receptor tyrosine kinases (Merlino and Helman, 1999), or loss-of-function mutations that normally suppress Ras signaling, such as neurofibromin(Reed and Gutmann, 2001). The recent identification of germline mutations of $H-R A S$ in Costello syndrome, a genetic disorder that predisposes affected individuals to embryonic tumors including embryonal rhabdomyosarcoma, further validates the role of H-Ras as an important genetic mutation in the development of rhabdomyosarcoma(Aoki et al., 2005). Following a detailed explanation of the techniques used to transform skeletal muscle cells, there will be a description of the assays used to verify expression of the transgenes introduced into the cell lines, and the methods used to evaluate their tumorigenic capacity.

\section{Materials and Methods}

\section{Overview}

This genetically defined model of rhabdomyosarcoma is based upon the serial stable introduction of the transgenes encoding SV40 DNA tumor virus early region, hTERT, and HRas ${ }^{\mathrm{G} 12 \mathrm{~V}}$ into primary human skeletal muscle myoblasts (HSMM cells) obtained from Lonza (formerly Cambrex Corp.), using amphotrophic retrovirus. The resulting transformed cells are abbreviated as "HSMM T-H-R" cells. It is important to note that although another type of primary human skeletal muscle cell (SkMC cells, Lonza catalog number CC-2561) is available from this supplier, when transformed by the T-H-R approach these cells yield tumors of varying phenotype, suggesting that the stock culture of primary SkMC cells is a heterogeneous population that can not reproducibly generate tumors resembling rhabdomyosarcoma. HSMM cells are therefore recommended. Because the plasmids and methodology used to generate infectious amphotrophic retrovirus are as published previously (O'Hayer and Counter, 2005), when the necessary reagents or methods are divergent from those published, the precise alternative approach is detailed.

\section{Creating HSMM T-H-R cells \\ Materials}

- Low passage HSMM cells confirmed by the supplier to be of skeletal muscle origin, as assessed by desmin staining $90 \%$ or greater, and capable of forming skeletal muscle myotubes when cultured in differentiation media (Lonza catalog number CC-2580)

- SKBM-2 media bullet kit (Lonza catalog number CC-3245)

- Subculturing reagent pack including HEPES-buffered saline solution, trypsin/EDTA, and trypsin-neutralizing solution (Lonza catalog number CC-5034) 
- Monoclonal antibodies to desmin (DakoCytomation catalog number M0760) and skeletal muscle-specific actin (DakoCytomation catalog number M0635, clone HHF35)

- Amphotrophic retrovirus encoding neomycin-SV40 early region (T/t-Ag), hygromycin-hTERT and puromycin-FLAG epitope-tagged-H-Ras ${ }^{G 12 V}$ (O'Hayer and Counter, 2005)

- Neomycin, diluted to stock $50 \mathrm{mg} / \mathrm{ml}$ (Gibco catalog number 10131-035)

- Hygromycin B, diluted to stock $50 \mathrm{mg} / \mathrm{ml}$ (Invitrogen catalog number 10687-010)

- Puromycin, reconstituted in $1 \times$ PBS to stock $1 \mathrm{mg} / \mathrm{ml}$ (Sigma catalog number P8833)

- Tissue culture plastic ware

\section{Methods}

a. Thaw, count, and seed HSMM cells into 6-cm tissue culture dishes per supplier instructions; allow cells to attach overnight. Subculture as suggested by the supplier.

b. Prior to beginning infection with amphotrophic retrovirus, verify expression of desmin and skeletal muscle-specific actin in the HSMM starting cell population by subjecting $100 \mu \mathrm{g}$ HSMM whole cell lysates to standard separation on SDS-PAGE and immunoblotting with anti-desmin monoclonal antibody at a dilution of 1:200, and anti-skeletal muscle specific actin antibody at a dilution of 1:200, followed by a secondary HRP-conjugated anti-mouse antibody and chemiluminescence exposure. On standard SDS-PAGE, desmin and skeletal muscle specific actin migrate at approximately 53 and $38 \mathrm{kD}$, respectively. In combination with a positive assay for myotube formation, expression of these protein markers verifies the skeletal muscle origin of the HSMM cells.

c. To begin the process of retroviral infection, when HSMM cells are $40 \%$ confluent, aspirate SKMB-2 media and replace with media containing amphotrophic retrovirus encoding SV40 T/t antigen. Amphotrophic virus will have been made in DMEM with $10 \%$ FBS; HSMM cells will tolerate a 24-hour exposure to this media. See (O'Hayer and Counter, 2005) on the preparation of amphotrophic retrovirus and related safety measures when working with this method of transgene delivery.

d. After 24 hours, remove viral media and replace with SKBM-2. Washing is not necessary.

e. After 24 hours, initiate antibiotic selection for neomycin-expressing HSMM cells, indicating that they have incorporated the retrovirus, by adding neomycin to working concentration of $250 \mu \mathrm{g} / \mathrm{ml}$. Selection should take at maximum 10 days. If there is excessive cell death, replace with fresh neomycin selection media so that dead or dying cells do not interfere with the growth of the remaining adherent cells. Once the HSMM cells are stably expressing the SV40 early region, they will be capable of bypassing tissue-culture induced senescence, and will be amenable to further transduction with hTERT and H-Ras ${ }^{\mathrm{G} 12 \mathrm{~V}}$. If the HSMM cells become more than $70 \%$ confluent during selection process, subculture using the reagent pack as per supplier instructions so that cells maintain log phase growth. There will be a very low background rate of spontaneous differentiation to myotubes if this practice is followed.

f. In a similar manner to the SV40 early region transgene, transduce cells with amphotrophic retrovirus encoding hTERT, using hygromycin B at a working concentration of $50 \mu \mathrm{g} / \mathrm{m}$ for 10 days, and then amphotrophic retrovirus encoding 
FLAG-H-Ras ${ }^{\mathrm{G} 12 \mathrm{~V}}$, using puromycin at a working concentration of $0.25 \mu \mathrm{g} / \mathrm{ml}$ for 7 days. Because HSMM cells will not tolerate culturing in more than one selection antibiotic at a time, perform each retroviral infection and its associated selection separately. See (O'Hayer and Counter, 2005) on the preparation of these amphotrophic retrovirus.

g. Following the introduction of each transgene, cells surviving selection should be frozen per supplier instructions and stored in liquid nitrogen so that future genetic manipulations can be performed as needed. Minimal cell passages should occur between transgene introductions.

h. Following final infection and selection, confirm that the HSMM T-H-R cells do not shed amphotrophic retrovirus, using the horizontal spread assay (Bielicki et al., 1996). Once confirmed, the cells may be manipulated outside of a BSL-2 facility.

\section{Validating transgene expression}

Prior to using the HSMM T-H-R cells for experimental inquiry, it is critical to examine the expression level of each of the introduced transgenes (Linardic et al., 2005). To prove stable expression of the SV40 early region, separate $100 \mu \mathrm{g}$ of an HSMM T-H-R whole cell lysate by standard SDS-PAGE and immunoblot using a monoclonal antibody against large $\mathrm{T}$ antigen (Santa Cruz catalog number SC-147) at a dilution of 1:1000, followed by a secondary HRPconjugated anti-mouse antibody and chemiluminescence exposure. To prove expression of FLAG-epitope-tagged H-Ras ${ }^{\mathrm{G} 12 \mathrm{~V}}$, similarly separate and immunoblot an HSMM T-H-R whole cell lysate using anti-FLAG-M2 monoclonal antibody (Sigma catalog number F3165) at a dilution of 1:4000). SV40 large T antigen and H-Ras migrate at 94 and $21 \mathrm{kD}$, respectively.

Since hTERT will not be detectable at the protein level, use RT-PCR to confirm expression of the hTERT cDNA(Kendall et al., 2005). In brief, following the isolation of total RNA from HSMM T-H-R cells using the RNAzol B (TEL-TEST catalog number CS-104) or similar reagent, reverse transcribe $2 \mu \mathrm{g}$ of total RNA using the Omniscript RT (Qiagen catalog number 205111) or similar kit with OligodT (Life Technologies Invitrogen catalog number 18418-012) primer. Input $4 \mu \mathrm{l}$ of each reaction into PCR amplification reactions for hTERT cloned into a pBABE plasmid (5'-GAGGTGCAGAGCGACTAC and 5'-

GCTGTTCACCTGCAAATCCA), or GAPDH (5'-GAGAGACCCTCACTGCTG and 5'GATGGTACATGACAAGGTGC). Separate each PCR reaction on a standard $\%$ agarose gel.

Retroviral transduction may yield variable levels of stable transgene expression in target cells, depending upon the efficiency of retroviral production and infection. In this regard, a low level of FLAG-H-Ras ${ }^{\mathrm{G} 12 \mathrm{~V}}$ protein expression within the HSMM T-H-R cells will result in decreased tumorigenic potential (see next section for a discussion of the assays used to assess tumorigenic potential). That is, soft agar colony count will be low and tumorigenesis in vivo will be slow. Therefore if needed, the HSMM T-H-R cells can be re-infected with and selected for retroviraltransduced FLAG-H-Ras ${ }^{\mathrm{G} 12 \mathrm{~V}}$ expression. Once the level of transgene expression has been examined and is satisfactory, further culturing of the HSMM T-H-R cell lines in selection antibiotics to promote transgene retention is not necessary, since the combination of introduced transgenes is not only tolerated in HSMM cells, but confers a growth advantage.

\section{Soft Agar and tumorigenic growth assays}

The soft agar assay is an in vitro method to measure cellular transformation, as assessed by the ability of cells to grow under anchorage-independent conditions. HSMM T-H-R cells are tested for anchorage-independent growth as previously described (O'Hayer and Counter, 2005), with the following modifications: 
a. HSMM T-H-R cells can be assayed in $2 \times \mathrm{SKBM}-2$, or to reduce cost, convert them to RPMI-1640 (or DMEM) with 10\% FBS, as most human rhabdomyosarcoma cell lines are grown in these common media types.

b. Count colonies after 4 , rather than 3 , weeks.

Although the soft agar assay measures anchorage-independent growth, a more rigorous assessment of tumorigenesis is to assay whether or not cells can form tumors in vivo. To determine whether the transformed HSMM T-H-R cells possess this property, these cells are injected as subcutaneous xenografts in immunocompromised SCID-beige mice in vivo as previously described (O'Hayer and Counter, 2005), with the following modifications:

a. 7 million, rather than 10 million cells, is adequate for subcutaneous injection.

b. When preparing cells for injection, HSMM T-H-R cells require additional rinsing with PBS in order to effectively trypsinize them off of the tissue culture plate. Therefore, rinse each 15-cm dish of confluent HSMM T-H-R cells twice with $10 \mathrm{ml}$ of $1 \times$ PBS, then trypsinize with $4 \mathrm{ml}$ of trypsin-EDTA and neutralize with $4 \mathrm{ml}$ trypsin-neutralizing solution.

c. Prior to injection into SCID-beige mice, cell lines should be tested to prove that they are bacteria, fungus, and mycoplasma-free. Bacterial and fungal contamination can be detected by visual inspection, but mycoplasma infection may be occult and therefore requires specific testing, such as with the Gen-Probe nucleic acid hybridization mycoplasma detection kit (Fisher Scientific catalog number GP-1591).

\section{Verification of tumor xenograft pathology characteristics}

To verify that the tumors generated from subcutaneous injection of HSMM cells transformed with T-H-R are appropriately phenocopying embryonal rhabdomyosarcoma, it is imperative to examine these tumors for morphology by standard $\mathrm{H} \& \mathrm{E}$ methods, and immunohistochemical markers of skeletal muscle. Harvested tumors are fixed in formalin, then placed in $75 \%$ ethanol pending embedding in paraffin. The first tier of markers traditionally used to immunohistochemically identify rhabdomyosarcoma includes desmin, myoglobin, and skeletal-muscle specific actin. Recently a second tier of markers, the muscle-specific transcription factors myogenin and MyoD1, was incorporated into the diagnostic algorithm, although the number of institutions that have developed these assays into high throughput mode is low. A description of the immunohistochemical techniques used to perform these assays is beyond the scope of this chapter, but we refer the reader to (Linardic et al., 2005; Morotti et al., 2006). For further proof of skeletal muscle origin, transmission electron microsocopy can be performed on the tumor xenografts to verify the presence of skeletal-muscle specific myofilaments (Linardic et al., 2005). A pathologist with experience in the analysis of soft tissue sarcomas should be involved in the interpretation of all of these analyses.

\section{Concluding Remarks}

To complement traditional cancer models employing tumor-derived cell lines and genetically manipulated mice, this method now provides a third approach in which to dissect the genetic events necessary to transform primary human skeletal muscle cells into cells that form embryonal rhabdomyosarcoma. The benefits of this method include its ability to flexibly evaluate the role of starting cell type in rhabdomyosarcoma histogenesis, and the role of specific genes in the discrete aspects of the pathology of rhabdomyosarcoma. Indeed, our exploration of two different starting primary human skeletal muscle cell types, SKMC and HSMM cells, illustrates the importance of choosing a cell type that is as homogeneous as possible, and once transformed accurately represents the tumor type being studied. In addition to its ability to accommodate a variety of cell types and genes, because this model is based on the step-wise 
accumulation of genes that initiate, as well propagate, the tumorigenic phenotype, it may be used to investigate both early and late events in rhabdomyosarcoma formation.

\section{References}

Aoki Y, Niihori T, Kawame H, Kurosawa K, Ohashi H, Tanaka Y, Filocamo M, Kato K, Suzuki Y, Kure $\mathrm{S}$, Matsubara Y. Germline mutations in HRAS proto-oncogene cause Costello syndrome. Nat Genet 2005;37:1038-1040. [PubMed: 16170316]

Bielicki J, Hopwood JJ, Anson DS. Correction of Sanfilippo A skin fibroblasts by retroviral vectormediated gene transfer. Hum Gene Ther 1996;7:1965-1970. [PubMed: 8930656]

Hahn WC, Counter CM, Lundberg AS, Beijersbergen RL, Brooks MW, Weinberg RA. Creation of human tumour cells with defined genetic elements. Nature 1999;400:464-468. [PubMed: 10440377]

Hamad NM, Elconin JH, Karnoub AE, Bai W, Rich JN, Abraham RT, Der CJ, Counter CM. Distinct requirements for Ras oncogenesis in human versus mouse cells. Genes Dev 2002;16:2045-2057. [PubMed: 12183360]

Keller C, Arenkiel BR, Coffin CM, El Bardeesy N, DePinho RA, Capecchi MR. Alveolar rhabdomyosarcomas in conditional Pax3:Fkhr mice: cooperativity of Ink4a/ARF and Trp53 loss of function. Genes Dev 2004;18:2614-2626. [PubMed: 15489287]

Kendall SD, Linardic CM, Adam SJ, Counter CM. A network of genetic events sufficient to convert normal human cells to a tumorigenic state. Cancer Res 2005;65:9824-9828. [PubMed: 16267004]

Linardic CM, Downie DL, Qualman SJ, Bentley RC, Counter CM. Genetic modeling of human rhabdomyosarcoma. Cancer Res 2005;65:4490-4495. [PubMed: 15930263]

Merlino G, Helman LJ. Rhabdomyosarcoma--working out the pathways. Oncogene 1999;18:5340-5348. [PubMed: 10498887]

Morotti RA, Nicol KK, Parham DM, Teot LA, Moore J, Hayes J, Meyer W, Qualman SJ. An immunohistochemical algorithm to facilitate diagnosis and subtyping of rhabdomyosarcoma: the Children's Oncology Group experience. Am J Surg Pathol 2006;30:962-968. [PubMed: 16861966]

Nanni P, Nicoletti G, De Giovanni C, Croci S, Astolfi A, Landuzzi L, Di Carlo E, Iezzi M, Musiani P, Lollini PL. Development of rhabdomyosarcoma in HER-2/neu transgenic p53 mutant mice. Cancer Res 2003;63:2728-2732. [PubMed: 12782574]

O'Hayer, KM.; Counter, CM. A genetically defined normal somatic human cell system to study ras oncogenesis in vitro and in vivo. In: Channing, Der, editor. Methods Enzymol. Vol. 407. Elsevier Inc.; San Diego: 2005. p. 637-647.

Rangarajan A, Hong SJ, Gifford A, Weinberg RA. Species- and cell type-specific requirements for cellular transformation. Cancer Cell 2004;6:171-183. [PubMed: 15324700]

Reed N, Gutmann DH. Tumorigenesis in neurofibromatosis: new insights and potential therapies. Trends Mol Med 2001;7:157-162. [PubMed: 11286939]

Sharp R, Recio JA, Jhappan C, Otsuka T, Liu S, Yu Y, Liu W, Anver M, Navid F, Helman LJ, DePinho RA, Merlino G. Synergism between INK4a/ARF inactivation and aberrant HGF/SF signaling in rhabdomyosarcomagenesis. Nat Med 2002;8:1276-1280. [PubMed: 12368906]

Tsumura H, Yoshida T, Saito H, Imanaka-Yoshida K, Suzuki N. Cooperation of oncogenic K-ras and p53 deficiency in pleomorphic rhabdomyosarcoma development in adult mice. Oncogene 2006;25:7673-7679. [PubMed: 16785989]

Xia SJ, Pressey JG, Barr FG. Molecular pathogenesis of rhabdomyosarcoma. Cancer Biol Ther 2002;1:97-104. [PubMed: 12170781]

Yeh E, Cunningham M, Arnold H, Chasse D, Monteith T, Ivaldi G, Hahn WC, Stukenberg PT, Shenolikar S, Uchida T, Counter CM, Nevins JR, Means AR, Sears R. A signalling pathway controlling c-Myc degradation that impacts oncogenic transformation of human cells. Nat Cell Biol 2004;6:308-318. [PubMed: 15048125] 